\title{
Protective effect of Opuntia ficus-indica fruits bread on streptozotocin-induced oxidative stress in rats
}

\author{
J. Y. Moon ${ }^{1}$, D. S. Kim ${ }^{1}$ and J. W. Choi ${ }^{2}$ \\ ${ }^{1}$ Department of Food Science and Biotechnology and ${ }^{2}$ College of Pharmacy, University of Kyungsung, Busan of Korea
}

Diabetes Mellitus is group of metabolic disorders characterized by hyperglycemia and abnormalities in carbohydrate, fat, and protein metabolism resulted from defects in insulin secretion, insulin sensitivity, or both ${ }^{(1)}$. Excess generation of highly reactive free radicals largely owing to hyperglycemia ${ }^{(2,3)}$ causes oxidative stress that may exacerbate the diabetes and its complications ${ }^{(4)}$. Opuntia ficus-indica var. Saboten (OFS) leaves and fruits have been of great interest as a treatment for arteriosclerosis, diabetes, gastritis, and hyperglycemia ${ }^{(5)}$. The goal of this study is designed to develop functional breads using Opuntia ficus-indica fruits powder (OFP) and to evaluate the protective effect of OFP bread (OFB) against oxidative damage in Streptozotocin (STZ)-treated diabetic rats.

OFB was made with adding $1 \%, 3 \%$ and $5 \%$ OFP to standard bread ingredients. Groups of male Sprague-Dawley rats ( $n 5$ in each) weighing 200 230 g were randomly assigned to five STZ (45 mg/kg, ip) induced diabetic groups; diabetic control group, OFB group (OFP $1 \%, 3 \%, 5 \%$ ) and reference group (glimepiride $5 \mathrm{mg} / \mathrm{kg}$, po). After six weeks with experimental diet, we evaluated malondialdehyde concentration, SOD, CAT, GPx activities and GSH level in the kidneys and the blood glucose levels taken after fasting (12 hour) to investigate the anti-diabetic effect of OFB. Serum was analyzed for BUN and AST and ALT.

Our results showed that OFB significantly reduced the blood glucose level and restored the body weight loss of diabetic rats (Fig. 1; $P<0.05)$. The supply of OFB reduced serum AST and ALT. BUN was also diminished by the OFB supplementation which implied an improvement of renal damage (Fig. 2; $P<0.05$ ). The malondialdehyde concentration in the kidney was significantly decreased by the OFB supplementation (Fig. 3; $P<0.05$ ). SOD, CAT, GPx activity and GSH concentrations in the STZ-induced diabetic rats were increased during the OFB supplementation. The results of our study concluded that OFB may have the antioxidative effect on STZ-induced oxidative stress in rats.

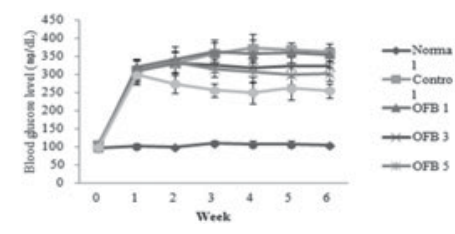

Fig. 1. Blood glucose level.

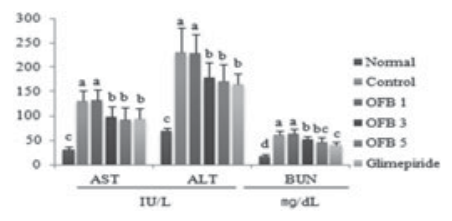

Fig. 2. Serum aminotransferase and BUN

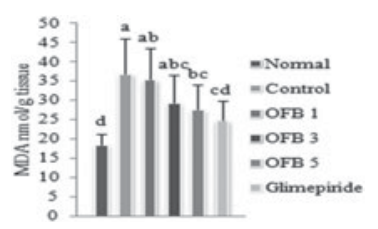

Fig. 3. Renal lipid peroxidation contents.

Table 1. Renal antioxidant enzymes activities

\begin{tabular}{|c|c|c|c|c|c|c|}
\hline \multirow[b]{2}{*}{ Group } & \multicolumn{2}{|c|}{ SOD $(\mathrm{U} / \mathrm{mg}$ protein/min) } & \multicolumn{2}{|c|}{ CAT (hydrogen peroxide nmole/mg protein/min) } & \multicolumn{2}{|c|}{ GPx (oxidized NADPH nmole/mg protein/min) } \\
\hline & Mean & SD & Mean & SD & Mean & SD \\
\hline Normal & 19.4 & 0.98 & 1.93 & 0.37 & 2.85 & 0.43 \\
\hline Control & 4.8 & 0.33 & 1.05 & 0.13 & 1.77 & 0.19 \\
\hline OFB 1 & 5.6 & 0.27 & 1.26 & 0.26 & 1.93 & 0.21 \\
\hline OFB 3 & 8.7 & 0.45 & 1.37 & 0.18 & 2.36 & 0.18 \\
\hline OFB 5 & 10.3 & 0.28 & 1.43 & 0.13 & 2.57 & 0.22 \\
\hline Glimepiride & 15.9 & 0.38 & 1.58 & 0.25 & 2.45 & 0.39 \\
\hline
\end{tabular}

Values are mean and standard deviation for three independent experiments. Data followed by different superscript are significant by Duncan new multiple range test from normal: $* P<0.05$.

1. Wells BG, Dipiro JT, Schwinghammer TL, Hamilton CW (2003) Pharmacotherapy Handbook 170-181.

2. West IC (2000) Diabetes Medicine 17, 171-180.

3. Johansen JS, Harris AK, Rychly DJ, Ergul A (2005) Cardiovascular Diabetology 4, 5.

4. Vinik AI \& Vinik E (2003) Am J Manag Care 9(3): 63-80.

5. El Kossori RL, Villaume C, Boustani EEl, Sauvaire Y \& Mejean L (1998) Plant Foods for Human Nutrition 52, $263-270$. 